\title{
Recovery of Presynaptic Dopaminergic Functioning in Rats Treated with Neurotoxic Doses of Methamphetamine
}

\author{
Wayne A. Cass and Michael W. Manning \\ Department of Anatomy and Neurobiology, University of Kentucky College of Medicine, Lexington, Kentucky 40536
}

Repeated administration of methamphetamine (METH) to animals can result in long-lasting decreases in striatal dopamine (DA) content. In addition, the evoked overflow of striatal DA is reduced in rats 1 week after neurotoxic doses of METH. However, whether these functional changes in DA release are permanent or tend to recover over time has not been established. In the present study we used in vivo electrochemistry and microdialysis to examine evoked overflow of DA in the striatum of METH-treated rats at several time points after treatment to determine if DA overflow would spontaneously recover. Male Fischer-344 rats were administered METH (5 mg/kg, s.c.) or saline four times in one day at $2 \mathrm{hr}$ intervals. In vivo electrochemistry experiments in anesthetized rats, and in vivo microdialysis studies in awake rats, were carried out 1 week, 1 month, 6 months, and 12 months after treatment. At 1 week after treatment there were significant decreases in potassiumand amphetamine-evoked overflow of DA, and in clearance of $\mathrm{DA}$, in the striatum of the METH-treated animals. Basal extracellular levels of DA and its metabolites were also decreased. Evoked overflow had partially recovered by 1 month. By 6 months evoked overflow of DA appeared to be normal in the METH-treated rats. However, whole tissue levels of striatal DA were still significantly decreased. All parameters were back to control values by 12 months. These results suggest that presynaptic dopaminergic functioning can recover to normal levels in the striatum of METH-treated rats by 12 months after treatment.

Key words: methamphetamine; neurotoxicity; striatum; dopamine; in vivo electrochemistry; in vivo microdialysis
Methamphetamine (METH), when given to animals in multiple smaller doses or a single large dose, can produce long-lasting changes in dopaminergic terminals in the striatum. These changes include decreases in dopamine (DA) content, DA release, DA uptake and tyrosine hydroxylase (TH) activity (Seiden and Ricaurte, 1987; Eisch et al., 1992; Gibb et al., 1994; Cass, 1997; Fukumura et al., 1998). Several studies have also indicated the presence of nerve terminal degeneration and reactive gliosis in the striatum after METH treatment (Lorez, 1981; Ricaurte et al., 1982; Bowyer et al., 1994; O'Callaghan and Miller, 1994; Pu and Vorhees, 1995), suggesting that degeneration of DA terminals is involved with the neurotoxic effects of METH. The time course for METH-induced changes in striatal DA systems is relatively long-lasting. In rats, striatal DA levels have been reported to be decreased at 6 months (Bittner et al., 1981) and $237 \mathrm{~d}$ (Friedman et al., 1998) after treatment. Similarly, in monkeys there are reductions in striatal DA levels up to 6 months after METH treatment (Seiden et al., 1976; Finnegan et al., 1982; Ando et al., 1985). One study reported a substantial depletion of striatal DA content in monkeys 4 years after treatment (Woolverton et al., 1989). Thus, METH-induced changes in striatal DA levels in animals appear to be long-lasting and in some cases may be permanent.

Recent reports have also indicated that METH may have neurotoxic effects in humans. Postmortem levels of DA, TH, and the DA transporter are decreased in striatum from chronic

Received April 1, 1999; revised June 3, 1999; accepted June 14, 1999.

This work was supported in part by United States Public Health Service Grant DA 10115. We thank Sherry L. Bailey for her technical assistance.

Correspondence should be addressed to Dr. Wayne A. Cass, Department of Anatomy and Neurobiology, MN 224 Chandler Medical Center, University of Kentucky, Lexington, KY 40536-0298.

Copyright (C) 1999 Society for Neuroscience 0270-6474/99/197653-08\$05.00/0
METH users (Wilson et al., 1996). However, in the same study, levels of DOPA decarboxylase and the vesicular monoamine transporter were not significantly decreased. This could indicate that the reductions in striatal dopaminergic markers in the METH users were caused by METH-induced downregulation and not terminal degeneration. However, in a more recent report, the density of DA transporters, as measured by positron emission tomography in living subjects, was reduced in abstinent METH abusers (McCann et al., 1998), suggesting a long-term loss of DA transporters or terminals. The possibility that there may be longterm or permanent changes in central DA systems in humans that abuse METH may impact whether these individuals later develop other neurological or neuropsychiatric disorders, such as Parkinson's disease, that involve central dopaminergic systems.

The purpose of the present study was to determine whether DA release and uptake in the striatum of METH-treated rats would spontaneously recover over an extended period of time. In vivo electrochemistry in anesthetized rats was used to measure potassium-evoked overflow of DA and clearance of DA in the striatum of rats at 1 week, and 1, 6, and 12 months after METH. In vivo microdialysis in awake rats was used to evaluate basal levels and potassium- and amphetamine-evoked overflow of DA and its primary metabolites, 3,4-dihydroxyphenylacetic acid (DOPAC) and homovanillic acid (HVA), at the same time points. Tissue levels of DA in the striatum were determined at the conclusion of each experiment to further evaluate the extent of dopaminergic recovery.

\section{MATERIALS AND METHODS}

Animals. Male Fischer-344 rats (Harlan Sprague Dawley, Indianapolis, IN) weighing 230-265 gm at the start of the study were used for these experiments. They were housed in groups of two under a $12 \mathrm{hr}$ light/dark cycle with food and water available ad libitum. All animal use procedures 
were in strict accordance with the National Institutes of Health Guide for the Care and Use of Laboratory Animals and were approved by the Animal Care and Use Committee at the University of Kentucky.

Methamphetamine treatment. Rats were injected subcutaneously with 5 $\mathrm{mg} / \mathrm{kg} \mathrm{METH}-\mathrm{HCl}$ (Sigma, St. Louis, MO) in saline $(1 \mathrm{ml} / \mathrm{kg})$, or saline alone $(1 \mathrm{ml} / \mathrm{kg})$, four times in 1 day at $2 \mathrm{hr}$ intervals. The first injection of the series was given between 8:00 and 9:00 A.M., and the temperature of the room was $23-24^{\circ} \mathrm{C}$.

In vivo electrochemistry. One week, 1 month (28-32 d), 6 months (25-27 weeks), or 12 months (50-54 weeks) after the METH treatment the animals were anesthetized with urethane $(1.25-1.5 \mathrm{gm} / \mathrm{kg}$, i.p.), placed into a stereotaxic frame, and prepared for in vivo electrochemical recordings as previously described (Cass, 1996, 1997).

Nafion-coated single carbon fiber electrodes were constructed, calibrated in vitro, and attached to single-barrel micropipettes (Cass, 1996, 1997). The micropipettes had tip diameters of $10-20 \mu \mathrm{m}$, and their tips were $270-310 \mu \mathrm{m}$ from the tips of the electrodes. The micropipettes were filled with a solution containing $70 \mathrm{~mm} \mathrm{KCl}, 79 \mathrm{~mm} \mathrm{NaCl}$, and $2.5 \mathrm{~mm}$ $\mathrm{CaCl}_{2}, \mathrm{pH} 7.4$, or $200 \mu \mathrm{M}$ DA in $0.9 \% \mathrm{NaCl}$ with $100 \mu \mathrm{M}$ ascorbic acid added as an antioxidant, $\mathrm{pH} 7.4$.

Chronocoulometric electrochemical measurements were continuously made at $5 \mathrm{~Hz}$ and averaged to $1 \mathrm{~Hz}$ using an IVEC -10 system (Medical Systems Corporation, Greenvale, NY). The applied oxidation potential was $+0.55 \mathrm{~V}$ (vs $\mathrm{Ag} / \mathrm{AgCl}$ reference electrodes) for $100 \mathrm{msec}$, and the resting potential was $0.0 \mathrm{~V}$ for $100 \mathrm{msec}$. The oxidation and reduction currents were digitally integrated during the last $80 \mathrm{msec}$ of each 100 msec pulse. Electrode assemblies were initially positioned in the dorsal striatum (1.2-1.5 mm anterior to bregma, 2.2-2.3 $\mathrm{mm}$ lateral from midline, and $3.5 \mathrm{~mm}$ below the surface of the brain). For the potassiumevoked release studies, the baseline electrochemical signal was allowed to stabilize (5-10 min), and then $100-125 \mathrm{nl}$ of potassium solution $(70 \mathrm{~mm}$ $\mathrm{K}^{+}$) was applied by pressure ejection (Picospritzer II; General Valve, Fairfield, NJ) during the recordings to evoke the release of DA (Fig. $1 A$ ). The volume of fluid injected was determined by monitoring the amount of fluid displaced from the micropipette using a dissection microscope fitted with a reticule eyepiece. After the signal had returned to baseline, the electrode/micropipette assembly was lowered by $0.5 \mathrm{~mm}$. The new baseline was allowed to stabilize and then 100-125 $\mathrm{nl}$ of potassium solution was applied again. The potassium applications were repeated at $0.5 \mathrm{~mm}$ steps in order to map the striatum and nucleus accumbens (Fig. $1 B)$. A single recording pass was made in one hemisphere of each animal. The side of the pass was alternated between animals in each group.

Four animals in each group were used to examine the clearance of exogenously applied DA. These experiments were carried out as above with the exception that the micropipette contained a solution of $200 \mu \mathrm{M}$ DA (Cass and Gerhardt, 1994, 1995), and the recording pass was $0.5 \mathrm{~mm}$ anterior to bregma and $3.0 \mathrm{~mm}$ lateral from midline. The depth of the recordings was $3.5-6.0 \mathrm{~mm}$ below the surface of the brain. The amount of DA solution applied at each site was adjusted to achieve a signal of 1-5 $\mu \mathrm{M}$ in amplitude.

In vivo microdialysis. Four or five days (animals in the 1 week group), or $7 \mathrm{~d}$ (animals in the 1 month, 6 month, and 12 month groups) before their scheduled microdialysis experiments the rats were anesthetized with sodium pentobarbital $(50 \mathrm{mg} / \mathrm{kg}$, i.p.) and positioned in a stereotaxic frame. All surgery was performed using sterile instruments and aseptic conditions. The skull was exposed, and a small hole was drilled in the skull over the right striatum. A guide cannula was lowered into the brain so that its tip was positioned $1.2 \mathrm{~mm}$ anterior to bregma, $2.5 \mathrm{~mm}$ right from midline, and $3.5 \mathrm{~mm}$ below the surface of the cortex. The cannula was secured to the skull with anchoring screws and dental acrylic. The incision was closed with sutures and Vetbond tissue adhesive $(3 \mathrm{M}$, St. Paul, MN), and the animals were placed in round microdialysis bowls and allowed to recover. One to two days before the dialysis experiments the rats were tethered to the microdialysis system (BeeKeeper system; BAS Inc., West Lafayette, IN), without dialysis probes in place, for 4-8 hr to help them acclimate to the system.

On the morning of the dialysis experiments the animals were connected to the dialysis system, and probes were inserted into the guides (CMA 12 probes, $3 \mathrm{~mm}$ long dialysis membrane; CMA/Microdialysis, Acton, MA). The probes were perfused with artificial CSF, $\mathrm{pH} 7.4$, containing (in mM): $145 \mathrm{NaCl}, 2.7 \mathrm{KCl}, 1.2 \mathrm{CaCl}_{2}, 1.0 \mathrm{MgCl}_{2}, 0.2$ ascorbic acid, and $2.0 \mathrm{NaH}_{2} \mathrm{PO}_{4}$ (Moghaddam and Bunney, 1989). Flow rate was $1.2 \mu \mathrm{l} / \mathrm{min}$, and fractions of dialysate were collected at $20 \mathrm{~min}$ intervals. After a $3 \mathrm{hr}$ equilibration period and the collection of three baseline fractions, the overflow of DA was stimulated locally. First the

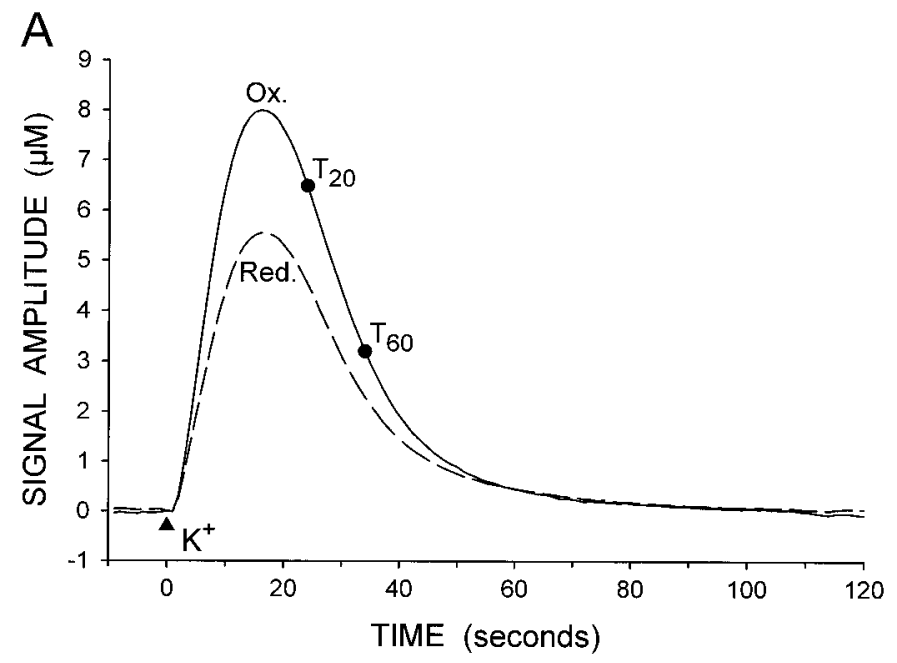

B

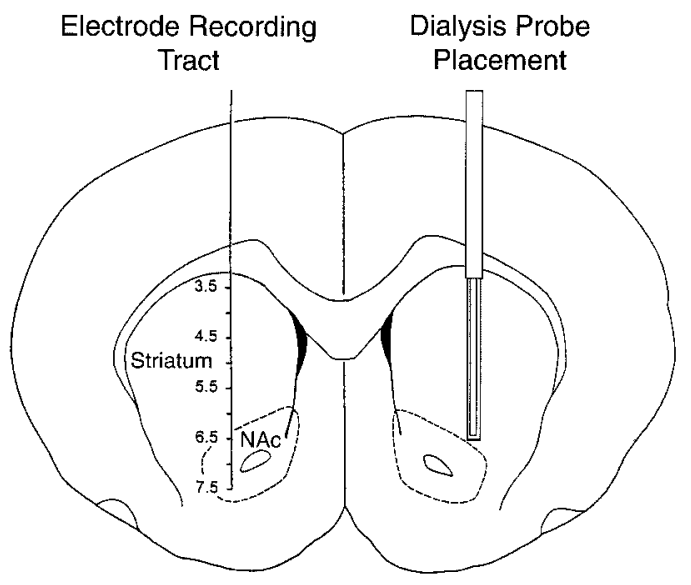

Figure 1. A, Potassium-evoked overflow of DA in the striatum of a control rat. Potassium $\left(125 \mathrm{nl}, 70 \mathrm{mM} \mathrm{K}^{+}\right)$was applied at the arrowhead at time 0 . The solid line shows the oxidation current response $(O x$.), and the dashed line is the corresponding reduction current response (Red.). The reduction/oxidation current ratio (0.69) indicates that the predominate electroactive species detected was DA. The $T_{20}$ and $T_{60}$ time points used for calculating clearance rate are indicated on the oxidation curve. $B$, Illustration of the location of the recording sites for the in vivo electrochemical recordings and the positioning of the microdialysis probe for the dialysis experiments. For the electrochemistry studies, data were collected at $0.5 \mathrm{~mm}$ steps throughout the dorsoventral extent of the striatum and nucleus accumbens (NAc) on one side of the brain (3.5-7.5 $\mathrm{mm}$ below the surface of the cortex). In vivo electrochemistry and in vivo microdialysis were performed on separate groups of rats. This diagram represents a coronal section of the forebrain $\sim 1.2 \mathrm{~mm}$ anterior to bregma.

perfusate solution was switched to a $100 \mathrm{~mm} \mathrm{~K}^{+}$solution (in mM: 47.7 $\mathrm{NaCl}, 100 \mathrm{KCl}, 1.2 \mathrm{CaCl}_{2}, 1.0 \mathrm{MgCl}_{2}, 0.2$ ascorbic acid, and 2.0 $\mathrm{NaH}_{2} \mathrm{PO}_{4}, \mathrm{pH} 7.4$ ) for a single 20 min fraction and then switched back to the original perfusate for five additional fractions. Then D-amphetamine $(100 \mu \mathrm{M})$ was included in the perfusate for one $20 \mathrm{~min}$ fraction followed by five final fractions with normal artificial CSF. Dialysate samples were analyzed immediately for DA, DOPAC, and HVA by HPLC (Cass, 1997) or frozen on dry ice, stored at $-80^{\circ} \mathrm{C}$, and analyzed within 1 week.

Tissue collection and HPLC analysis. At the end of the experiments, the animals were killed by decapitation while still anesthetized with urethane (in vivo electrochemistry experiments) or after anesthetizing with $\mathrm{CO}_{2}$ (in vivo microdialysis experiments). The brains were rapidly removed and chilled in ice-cold saline. A coronal slice of brain $\sim 2$-mmthick at the level of the electrode or dialysis probe track was made with the aid of a chilled brain mold (Rodent Brain Matrix; ASI Instruments, Warren, MI). The location of each recording electrode or dialysis probe 

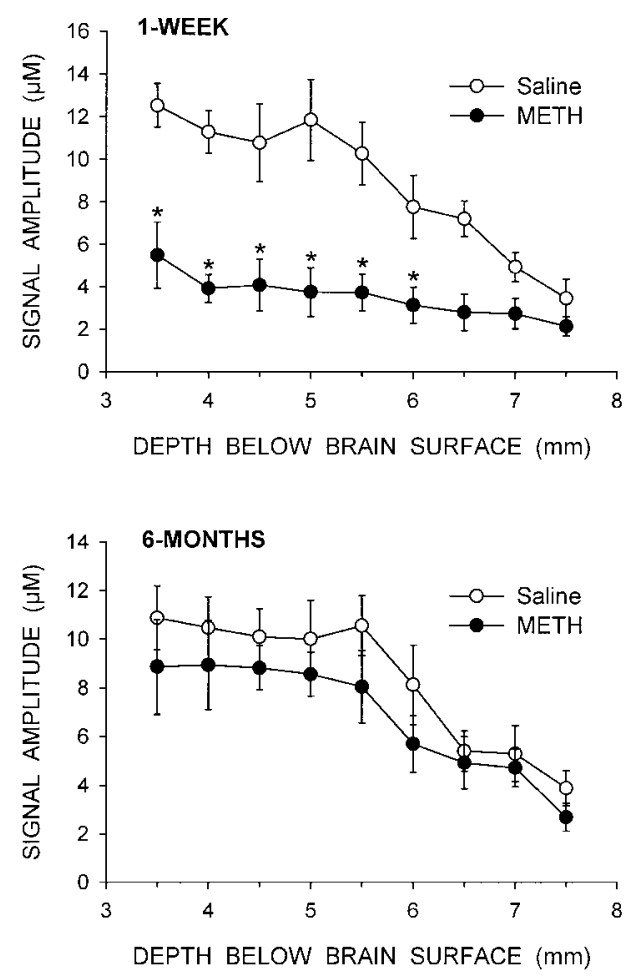
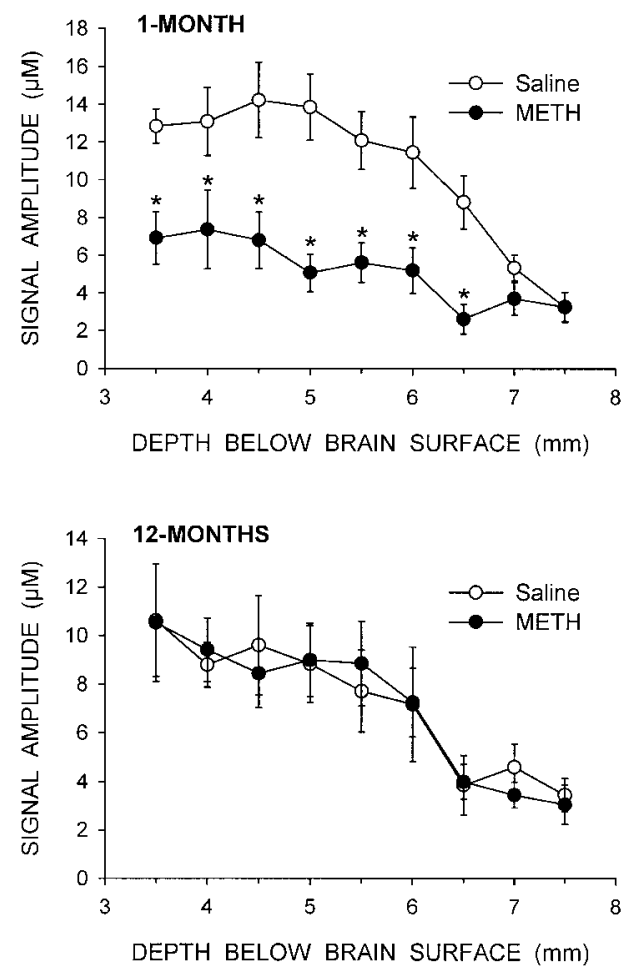

Figure 2. Summary of potassiumevoked DA signal amplitude throughout the striatum and nucleus accumbens of animals treated with saline or METH. Experiments were performed at 1 week, 1 month, 6 months, or 12 months after treatment with saline or METH, as indicated in the figure. The data shown are mean \pm SEM values for eight animals per group. ${ }^{*} p<0.05$ versus saline group at same depth (three-way repeated measures ANOVA followed by Newman-Keuls post hoc comparisons). was confirmed by noting blood left in the track or by later sectioning on a cryostat if the track was not easily visible. The striatum was dissected from that half of the brain contralateral to the electrode or dialysis probe track. The tissue was placed into a preweighed vial, weighed, and frozen on dry ice. Samples were stored at $-80^{\circ} \mathrm{C}$ until assayed by HPLC with electrochemical detection as previously described (Cass, 1996, 1997). Retention times of standards were used to identify peaks, and peak heights were used to calculate recovery of internal standard (dihydroxybenzylamine) and amount of DA and metabolites. Results were expressed as micrograms per gram wet weight of tissue.

Data analysis. The electrodes used in this study, although relatively insensitive to ascorbic acid because of the Nafion coating (Gerhardt et al., 1984), can still detect serotonin (5-HT) if the levels are high enough. In order to confirm that the responses detected were caused primarily by DA, both the reduction and oxidation currents were recorded, and the ratio of the reduction current to oxidation current was calculated for each $\mathrm{K}^{+}$-induced response. The electrodes used in this study exhibit reduction/oxidation current ratios of 0.4 or greater for DA, ratios of $0.1-0.2$ for 5-HT, and ratios of 0.0 for ascorbic acid (Luthman et al., 1993; Cass, 1997). All of the responses included in the results had reduction/oxidation ratios of at least 0.4 , indicating that DA was the predominant compound detected by the electrodes after potassium application. For statistical analysis of the electrochemistry data two parameters were analyzed: (1) the maximum amplitude of the signals resulting from the local application of potassium or DA; and (2) the clearance rate of the signals (the slope of the linear, declining portion of the signal between the $\mathrm{T}_{20}$ and $\mathrm{T}_{60}$ time points shown on Fig. $1 A$; the $\mathrm{T}_{20}$ and $\mathrm{T}_{60}$ points represent the time points at which the signal has declined by 20 and $60 \%$ of the maximum amplitude). The clearance rate is an estimate of the maximal slope of the declining phase of the signal and is related to the functioning of DA transporters in the region of the recording electrode (Wightman and Zimmerman, 1990; Cass et al., 1993; Suaud-Chagny et al., 1995; Garris et al., 1997). Dialysis data were expressed as concentration of DA in the dialysate. Basal levels were defined as the average value in the three fractions preceding stimulation by excess potassium. All probes were calibrated in vitro before use to determine acceptable probes (recovery of DA at least 15\%). However, values were not corrected for in vitro recoveries because uncorrected values may be better correlated to true values (Glick et al., 1994). Electrochemical data, microdialysis data, and tissue monoamine concentrations were analyzed using two-way or three-way ANOVA followed by Newman-Keuls post hoc comparisons as indicated in the results.

\section{RESULTS}

\section{In vivo electrochemistry}

Locally applied potassium produced signals with reduction/oxidation current ratios characteristic for DA at all recording sites in the striatum and nucleus accumbens of both the saline- and METH-treated animals. A representative response from the striatum of a saline-treated control animal is shown in Figure $1 \mathrm{~A}$. Recordings at a depth of 3.5-6.0 mm below the surface of the brain are contained within the striatum, whereas recordings at a depth of $6.5 \mathrm{~mm}$ are approximately at the junction of the striatum and nucleus accumbens (Fig. 1B). Recordings at depths of 7.0 and $7.5 \mathrm{~mm}$ are within the nucleus accumbens.

Signal amplitudes from the saline and METH groups at the four time points are shown in Figure 2. The data were analyzed using a three-way ANOVA with treatment and time after treatment as between factors, and depth of recording as a within factor. Significant main effects were found for treatment $(p<$ $0.001)$ and depth $(p<0.001)$. Significant interactions were found for treatment $\times$ time $(p<0.01)$, treatment $\times$ depth $(p<0.01)$, and treatment $\times$ time $\times$ depth $(p<0.05)$. The METH groups at 1 week and 1 month were different from their saline controls, and the METH group at 1 week was different from the METH groups at 6 and 12 months $(p<0.05$; Newman-Keuls post hoc comparisons). At 1 week after the treatment there were significant decreases in potassium-evoked overflow of DA in the dorsal striatum, but not nucleus accumbens, as described previously (Cass, 1996, 1997). The lack of METH-induced changes in evoked overflow of DA in the nucleus accumbens reflects the lower vulnerability of the nucleus accumbens to the DA-depleting effects of METH (Cass, 1997). By 1 month after treatment there was a tendency for signal amplitude to be higher in the METHtreated animals than amplitudes at 1 week, but the amplitudes were still significantly attenuated in the dorsal striatum compared to the saline controls. At 6 months there were no significant 

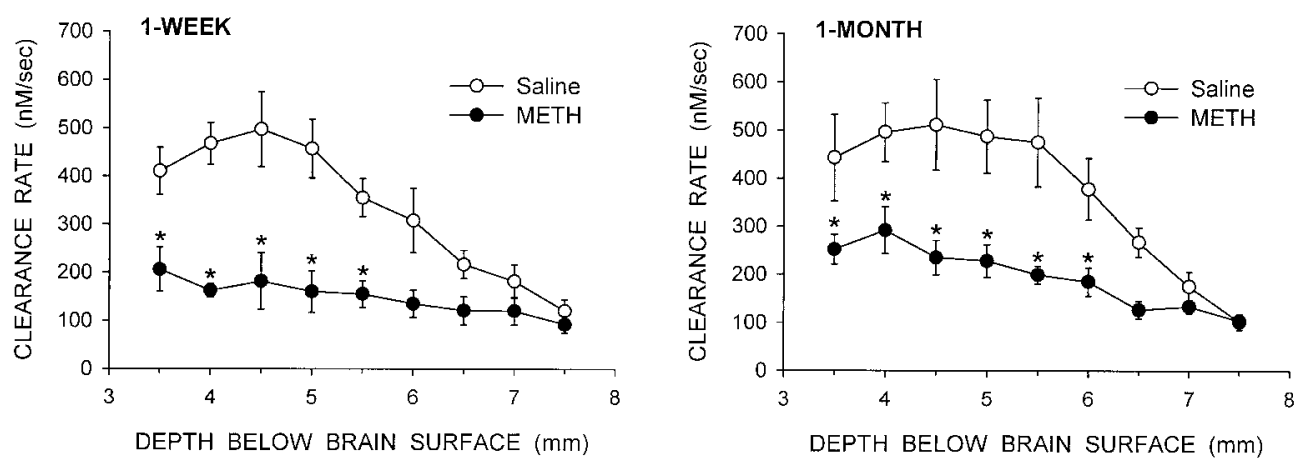

Figure 3. Summary of DA clearance rate after potassium application throughout the striatum and nucleus accumbens of animals treated with saline or METH. Experiments were performed at 1 week, 1 month, 6 months, or 12 months after treatment with saline or METH, as indicated in the figure. The data shown are mean \pm SEM values for eight animals per group. ${ }^{*} p<0.05$ versus saline group at same depth (threeway repeated measures ANOVA followed by Newman-Keuls post hoc comparisons).
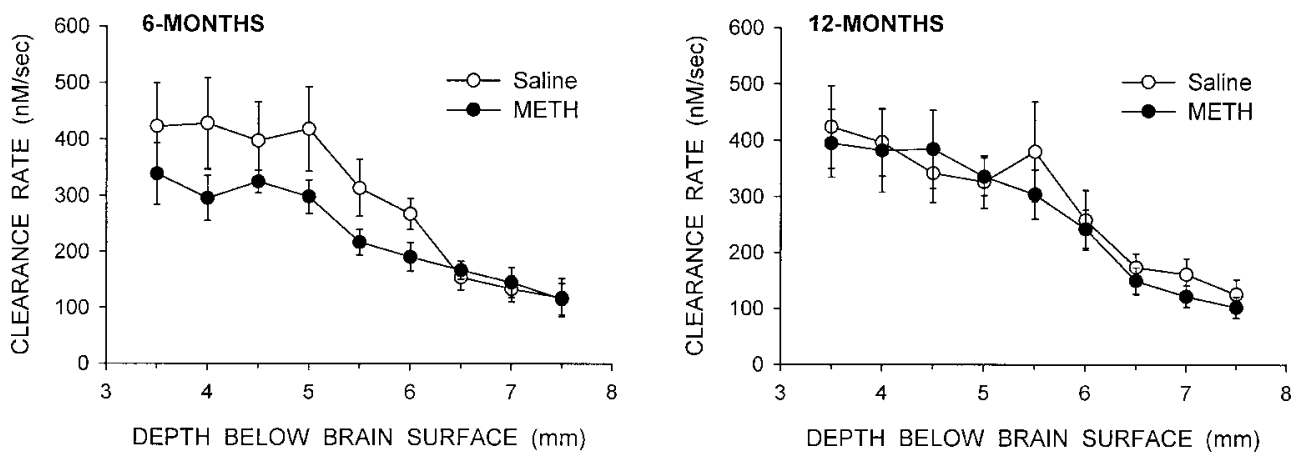

differences in signal amplitude between the saline and METH groups; however, there was a trend for lower amplitudes in the dorsal striatum of the METH-treated rats. By 12 months after treatment there were no differences between the saline- and METH-treated animals.

The patterns of changes in clearance rate were similar to the changes seen in signal amplitude (Fig. 3). A three-way repeated measures ANOVA indicated significant main effects for treatment $(p<0.001)$ and depth $(p<0.001)$, and significant interactions for treatment $\times$ time $(p<0.01)$, treatment $\times$ depth $(p<$ $0.001)$, and treatment $\times$ time $\times$ depth $(p<0.05)$. The METH groups at 1 week and 1 month were different from their saline controls, and the METH group at 1 week was different from the METH group at 12 months $(p<0.05$; Newman-Keuls post hoc comparisons). At 1 week and 1 month after treatment there were significant decreases in clearance rate in the dorsal striatum of the METH-treated animals compared to their controls (Fig. 3). However, at 6 and 12 months after treatment there were no significant differences in clearance rate at any depth between the saline and METH groups of animals.

For the experiments examining the clearance of exogenous DA, the amount of DA applied was adjusted to achieve signals with approximately the same amplitude in both the saline- and METH-treated rats. Only striatal sites were examined (depths of 3.5-6.0 mm below the surface of the brain), and for each rat the responses were averaged to get an overall mean for each animal. The signal amplitudes were $\sim 3.0 \mu \mathrm{M}$ for both the saline and METH groups at all four time points (Fig. 4). Clearance rates for these signals were decreased in the METH-treated animals by $57 \%$ in the 1 week group and $43 \%$ in the 1 month group. Clearance rates were not different between the saline- and METH-treated animals at either the 6 or 12 month time points.

\section{In vivo microdialysis}

The local application of potassium and amphetamine into the striatum through the dialysis probes produced substantial in- creases in the extracellular levels of DA in the saline- and METH-treated rats in all experimental groups (Fig. 5). For statistical analyses of evoked overflow, the data were separated into potassium-evoked overflow (20-120 min) and amphetamineevoked overflow (140-240 min.) The data were then analyzed using three-way ANOVA with treatment and time after treatment as between factors, and minutes for collection of dialysis sample as a within factor. For the potassium-evoked overflow, significant main effects were found for treatment, time after treatment, and minutes (all $p<0.001$ ). Significant interactions were found for treatment $\times$ minutes $(p<0.001)$, time after treatment $\times$ minutes $(p<0.001)$, and treatment $\times$ time after treatment $\times$ minutes $(p<0.05)$. For the amphetamine-evoked overflow, significant main effects were found for treatment $(p<0.001)$ and minutes $(p<0.001)$. Significant interactions were found for treatment $\times$ minutes $(p<0.001)$, time after treatment $\times$ minutes $(p<0.05)$, and treatment $\times$ time after treatment $\times$ minutes $(p<0.05)$. At both 1 week and 1 month after treatment, there were significant decreases in potassium- and amphetamine-evoked overflow of DA in the METH-treated animals compared to the saline-treated controls (Fig. 5). In the animals treated 6 months earlier with METH, there was a trend for decreases in both potassium- and amphetamine-evoked overflow compared to the saline-treated controls. At 12 months after treatment, there was no difference in evoked overflow of DA between the saline and METH groups, similar to the in vivo electrochemistry results.

Basal levels of DA, DOPAC, and HVA from the dialysis experiments are shown in Figure 6. In the METH-treated animals, basal extracellular levels of DA were decreased by $29 \%$ at 1 week after treatment but had recovered to control levels by 1 month. Basal DOPAC levels in the METH-treated rats were decreased by $48 \%$ at 1 week and $29 \%$ at 1 month compared to controls. Basal HVA levels were similarly decreased in the METH group by $42 \%$ at 1 week and $29 \%$ at 1 month. Basal levels 

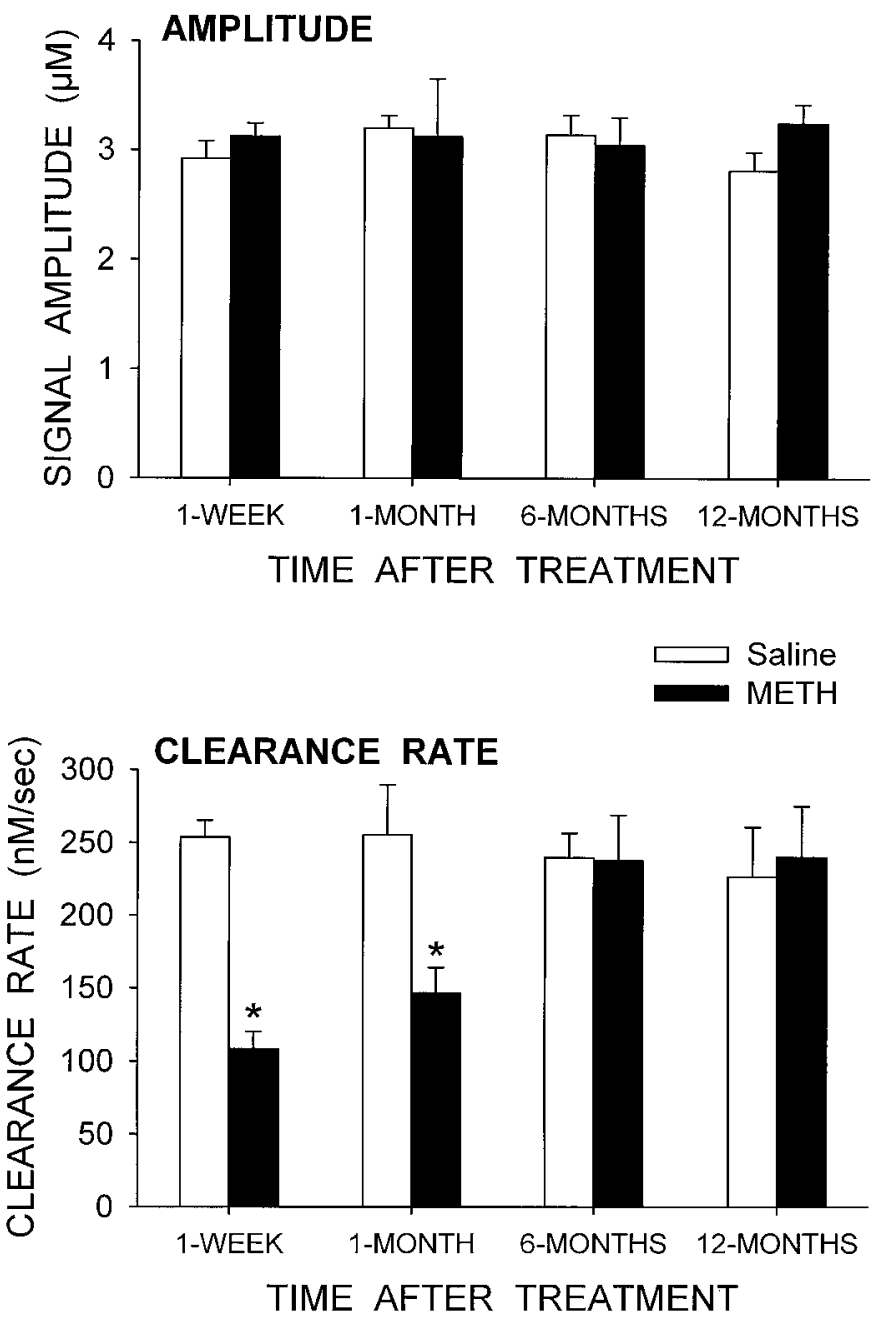

Figure 4. Clearance of exogenous DA in the striatum of saline- and METH-treated animals at four time points after treatment. DA was applied by pressure ejection into the striatum $(3.5-6.0 \mathrm{~mm}$ below the surface of the brain), and its clearance was monitored electrochemically. The amount of DA applied at each site was adjusted to achieve a signal of $\sim 3 \mu \mathrm{M}$ in amplitude (top graph). The corresponding clearance rates for the resulting signals are shown in the bottom graph. The data from all sites in each animal were averaged together to get a single mean value per animal. The values shown are mean \pm SEM for four animals per group. ${ }^{*} p<0.05$ versus saline group at same time (two-way ANOVA followed by Newman-Keuls post hoc comparisons).

of DOPAC and HVA at 6 and 12 months were not significantly different between the saline- and METH-treated animals.

\section{Tissue DA levels}

Postmortem levels of DA were not different between the animals used for the electrochemistry experiments and the microdialysis experiments, and the data were therefore grouped together. At 1 week after treatment, DA levels in the striatum were decreased by $54 \%$ in the METH-treated animals (Table 1). The decrease was $48 \%$ at 1 month and there was still a significant decrease of $20 \%$ at 6 months after treatment in the METH group. However, by 12 months there was no difference in striatal DA levels between the saline- and METH-treated animals. Tissue levels of DOPAC and HVA were also back to control values by 12 months after treatment (data not shown).

\section{DISCUSSION}

The present study was designed to evaluate whether METHinduced reductions in striatal DA release and content in rats would spontaneously recover over an extended period of time. Basal extracellular levels of DA, DOPAC, and HVA, potassiumand amphetamine-evoked overflow of DA, clearance of extracellular DA, and tissue content of DA all had returned to control levels by 6 months to 1 year after the METH treatment. All of the present results therefore indicate, that in the animal model used, presynaptic DA terminal functioning can completely recover by 12 months after treatment. However, it should be kept in mind that extent of recovery may depend on several factors, including species, because there are indications that some of the effects of METH may be permanent in nonhuman primates (Woolverton et al., 1989) and humans (McCann et al., 1998).

The changes found at 1 week after METH, decreases in basal levels of DA, DOPAC, and HVA, and reductions in potassiumand amphetamine-evoked overflow of DA, are similar to what we have reported previously for anesthetized and awake animals (Cass, 1997; Cass et al., 1998). In contrast to basal levels of DOPAC and HVA, basal levels of DA had returned to control levels by 1 month after the METH. This is similar to what has been reported in rats with partial 6-hydroxydopamine lesions of nigrostriatal DA neurons. Robinson et al. (1994) found that in rats with unilateral 6-hydroxydopamine lesions that reduced striatal DA levels by $\sim 52 \%$, microdialysate levels of striatal DA were significantly reduced at $4 \mathrm{~d}$ after the lesion but back to control levels by 3-4 weeks after lesion. Basal levels of DOPAC and HVA were reduced at both $4 \mathrm{~d}$ and 3-4 weeks. These results are similar to the present results at 1 week and 1 month after METH. The increase in basal levels of extracellular DA from 1 week to 1 month is likely caused by presynaptic adaptations that occur after lesions of nigrostriatal DA neurons in an attempt to normalize extracellular DA levels (Zigmond et al., 1990; Robinson et al., 1994). These adaptations could include factors such as an increase in the amount of DA released from remaining terminals as well as a decrease in the rate of removal of DA from the extracellular milieu caused by fewer DA transporters. Although basal levels of DA had recovered by 1 month after treatment, basal levels of DOPAC and HVA were still below control levels. In fact, recovery of basal extracellular levels of DOPAC and HVA followed more closely the recovery of tissue levels of DA, returning to control levels by 6 months after treatment when striatal DA levels had recovered to $80 \%$. These results support the hypothesis that basal extracellular levels of DOPAC and HVA are not caused solely by degradation of DA that is released from terminals, but rather may reflect to a large extent the total amount of synthesis and metabolism of DA occurring in surrounding DA terminals (Zetterström et al., 1988; Kuczenski and Segal, 1989; Cumming et al., 1992).

The recovery of potassium- and amphetamine-evoked overflow of DA also paralleled the recovery of tissue levels of DA. Thus, although basal extracellular levels of DA were back to normal by 1 month after METH, evoked overflow of DA did not recover until $\sim 6$ months after treatment. This suggests that by 1 month after METH, sufficient adaptations or recovery in releasable stores of DA have occurred to maintain normal basal release, but not stimulated release. The potassium and amphetamine stimulations we applied were likely close to maximal. Thus, the evoked overflow results may reflect the total amount of DA available in releasable pools. With lower stimulation intensities, evoked over- 

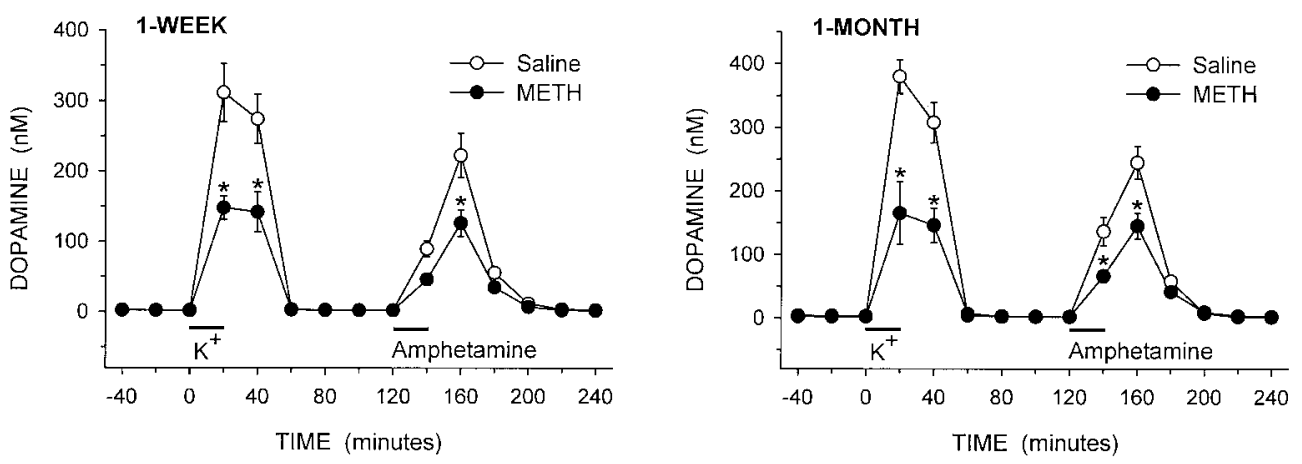

Figure 5. Dialysate levels of DA from the striatum of awake rats treated 1 week, 1 month, 6 months, or 12 months earlier with saline or METH. Excess potassium $(100 \mathrm{~mm})$ was included in the perfusate for $20 \mathrm{~min}$ starting at $0 \mathrm{~min}$ (horizontal bar above $K^{+}$), and $100 \mu \mathrm{M}$ amphetamine was included in the perfusate for $20 \mathrm{~min}$ starting at $120 \mathrm{~min}$ (horizontal bar above Amphetamine). The values shown are mean \pm SEM from six or seven animals in each group. $* p<0.05$ versus saline at same time point (three-way repeated measures ANOVA followed by Newman-Keuls post hoc comparisons).
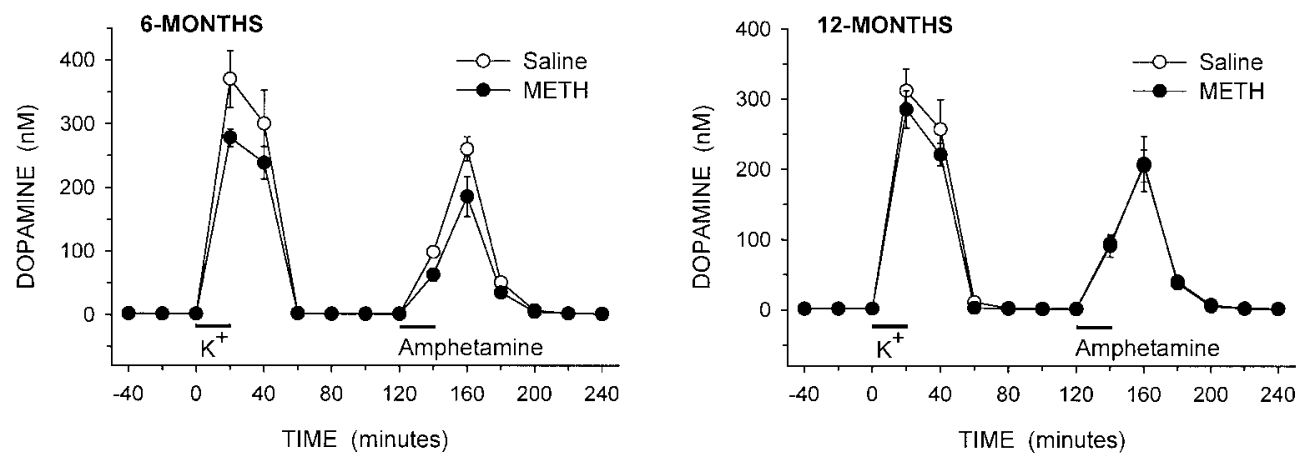

flow of DA may be normalized at an earlier time point. In addition, both the potassium- and amphetamine-evoked overflow of DA recovered at approximately the same rate, suggesting that the recovery or normalization processes that occur do not favor either calcium-dependent (potassium-evoked overflow) or calcium-independent (amphetamine-evoked overflow) release mechanisms.

The decrease in the clearance rate of endogenous DA observed in the METH-treated animals could be caused by a loss of functional DA transporters in the striatum. Alternatively, because the activity of the DA transporter can be modulated by D2-like DA receptors (Meiergerd et al., 1993; Parsons et al., 1993; Cass and Gerhardt, 1994; Dickinson et al., 1999), the decrease in clearance rate could be caused by METH-induced decreases in evoked overflow of DA. The higher extracellular DA concentrations achieved in the saline-treated animals after potassium stimulation may be increasing activity of the transporters in the striatum, whereas the converse may be occurring in the METHtreated animals. The experiments examining the clearance of exogenous DA were designed to examine this possibility. The amount of DA applied was adjusted to achieve signals of equal amplitude in the saline and METH-treated animals. If the decrease in clearance of endogenous DA was caused by a lower extracellular concentration of DA after the potassium application, then the clearance of exogenous DA should be similar in the saline and METH groups. However, there were still significant decreases in the clearance rate in the METH-treated animals at 1 week and 1 month after treatment. This suggests that the decrease in clearance rate is attributable, at least in part, to a loss of functional DA transporters after the METH treatment, and not just caused by D2-like receptor modulation of transporter activity.

Several others studies have examined the possibility of recovery of dopaminergic indices in animals treated with neurotoxic doses of METH or amphetamine. In rats, striatal DA levels recovered from an $\sim 70 \%$ depletion $48 \mathrm{~d}$ after METH to a $20 \%$ depletion at 237 d after METH (Friedman et al., 1998). In older rats, DA levels in the striatum increased from a loss of $\sim 78 \%$ at $14 \mathrm{~d}$ to $\sim 55 \%$ loss at 4 months after treatment with neurotoxic doses of amphetamine (Bowyer et al., 1998). In vervet monkeys treated with neurotoxic doses of METH or amphetamine, there is a partial recovery of striatal DA content by 12 weeks after treatment (Melega et al., 1997b), and a progressive improvement in fluoro-L-DOPA uptake that can return to pretreatment values by 24 months (Melega et al., 1997a). Thus, like the present results, these studies indicate that striatal dopaminergic systems can substantially recover from the effects of METH or amphetamine over an extended period of time. On the other hand, rhesus monkeys treated with a more extensive METH treatment can still have a substantial depletion of striatal DA content and DA uptake 4 years after treatment (Woolverton et al., 1989), and human METH abusers can have reductions in DA transporter density after 3 years of abstinence (McCann et al., 1998). These later studies suggest that some of the effects of METH on DA terminals may be permanent. It is likely that possible species differences in vulnerability to METH neurotoxicity, and extent and severity of METH treatment will play a significant role in the degree of recovery possible after METH treatment.

Although the present results indicate recovery of presynaptic dopaminergic processes in the striatum of our METH-treated animals, they do not identify the mechanism for this recovery. One possibility is that remaining DA axons are sprouting and reinnervating the striatum. Another possibility is that DA synthesis and release in remaining DA terminals are upregulated in a compensatory manner similar to that reported after 6-hydroxydopamine lesions (Zigmond et al., 1990; Robinson et al., 1994). Still a third possibility is that the changes observed in the METH-treated rats are the result of a transient downregulation of dopaminergic processes caused by the intense stimulation by METH. This latter explanation is unlikely to be the primary 

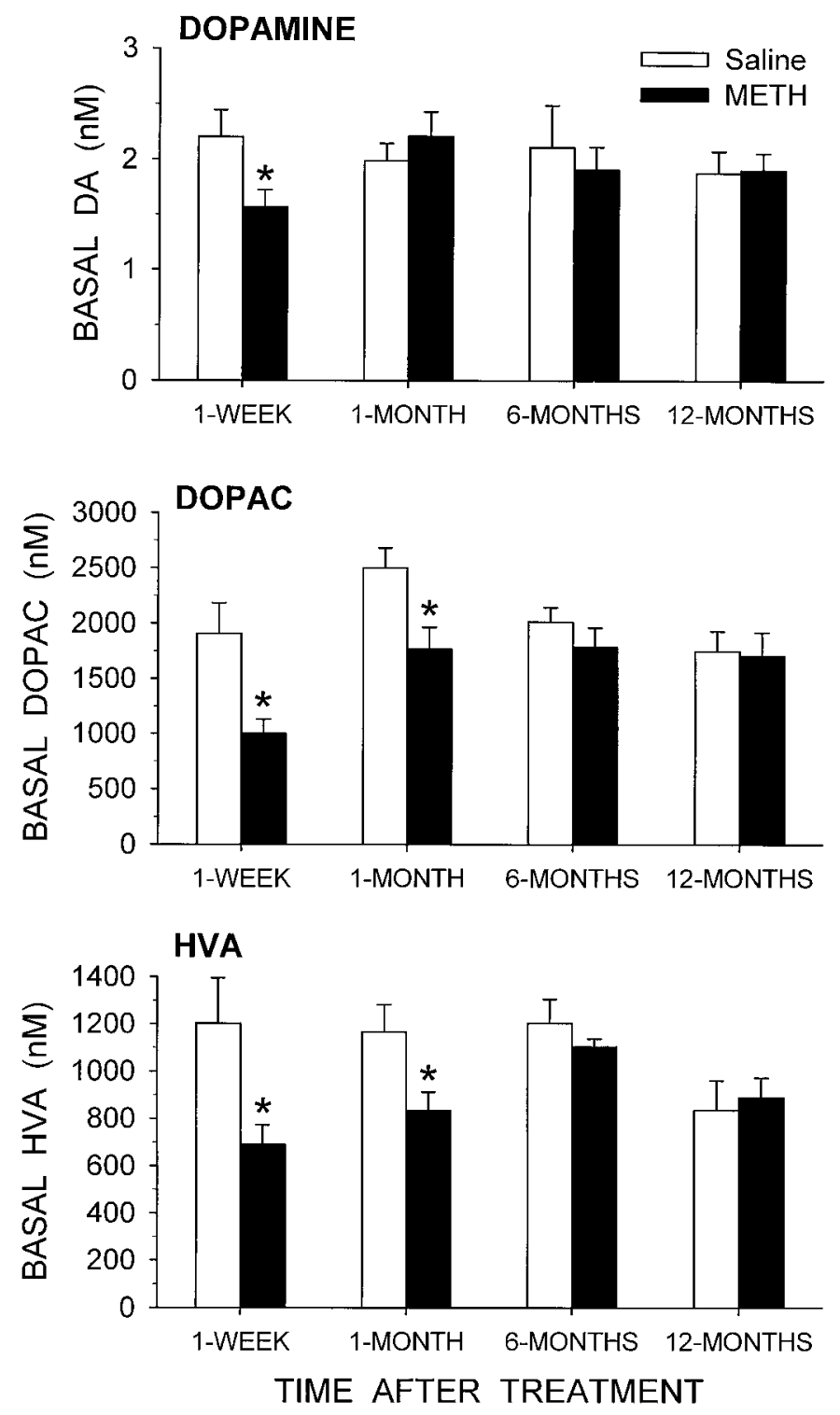

Figure 6. Basal levels of dialysate DA, DOPAC, and HVA from the striatum of awake rats treated 1 week, 1 month, 6 months, or 12 months earlier with saline or METH. The data shown are mean values \pm SEM from six or seven animals in each group. ${ }^{*} p \leq 0.05$ versus saline group at same time (two-way ANOVA followed by Newman-Keuls post hoc comparisons).

mechanism for several reasons. First, the time course for recovery, 6 months to 1 year, is substantially longer than would be expected if downregulation was entirely responsible. Second, silver degeneration staining and glial fibrillary acidic protein immunohistochemistry studies indicated the presence of degenerating axons and terminals, and reactive gliosis, in the striatum of separate groups of rats examined $2 \mathrm{~d}$ after saline or METH administration (our unpublished observations). This suggests that our METH treatments are producing degeneration of DA terminals in the striatum and not simply downregulating presynaptic dopaminergic processes. Third, 5-HT neurons appear to sprout and reinnervate forebrain target structures over an extended period of time in animals treated with neurotoxic doses of amphetamine derivatives (Axt et al., 1994). Similar processes may occur with DA axons in the striatum of METH-treated animals.
Table 1. Striatal levels of DA from animals treated with saline or METH

\begin{tabular}{lccll} 
& \multicolumn{4}{l}{ Time after treatment } \\
\cline { 2 - 5 } Treatment & 1 week & 1 month & 6 months & 12 months \\
\hline Saline & $13.74 \pm 0.66$ & $14.06 \pm 0.77$ & $13.52 \pm 0.58$ & $13.81 \pm 0.78$ \\
METH & $6.39 \pm 0.71^{*}$ & $7.25 \pm 0.61^{*}$ & $10.84 \pm 0.43^{*}$ & $12.70 \pm 0.77$
\end{tabular}

Levels are expressed as micrograms per gram wet weight of tissue. Values are mean \pm SEM for $14-15$ animals per group.

${ }^{*} p<0.05$ versus saline group at same time point (two-way ANOVA followed by Newman-Keuls post hoc comparisons).

Thus, the most likely explanation for the recovery reported in the present study is a combination of sprouting of remaining axons and upregulation of synthesis and release of DA in existing terminals. In addition, the possible induction of new TH-positive cells within the striatum of METH-treated animals, as demonstrated by Bowyer et al. (1998) in rats administered neurotoxic doses of amphetamine, could also play a role in the recovery. Further experiments will be necessary to positively identify the mechanisms responsible for this recovery.

\section{REFERENCES}

Ando K, Johanson CE, Seiden LS, Schuster CR (1985) Sensitivity changes to dopaminergic agents in fine motor control of rhesus monkeys after repeated methamphetamine administration. Pharmacol Biochem Behav 22:737-743.

Axt KJ, Mamounas LA, Molliver ME (1994) Structural features of amphetamine neurotoxicity in the brain. In: Amphetamine and its analogs: psychopharmacology, toxicology, and abuse (Cho AK, Segal DS, eds), pp 315-367. San Diego: Academic.

Bittner SE, Wagner GC, Aigner TG, Seiden LS (1981) Effects of highdose treatment of methamphetamine on caudate dopamine and anorexia in rats. Pharmacol Biochem Behav 14:481-486.

Bowyer JF, Davies DL, Schmued L, Broening HW, Newport GD, Slikker Jr W, Holson RR (1994) Further studies of the role of hyperthermia in methamphetamine neurotoxicity. J Pharmacol Exp Ther 268:1571-1580.

Bowyer JF, Frame LT, Clausing P, Nagamoto-Combs K, Osterhout CA, Sterling CR, Tank AW (1998) Long-term effects of amphetamine neurotoxicity on tyrosine hydroxylase mRNA and protein in aged rats. J Pharmacol Exp Ther 286:1074-1085.

Cass WA (1996) GDNF selectively protects dopamine neurons over serotonin neurons against the neurotoxic effects of methamphetamine. J Neurosci 16:8132-8139.

Cass WA (1997) Decreases in evoked overflow of dopamine in rat striatum after neurotoxic doses of methamphetamine. J Pharmacol Exp Ther 280:105-113.

Cass WA, Gerhardt GA (1994) Direct in vivo evidence that D2 dopamine receptors can modulate dopamine uptake. Neurosci Lett 176:259-263.

Cass WA, Gerhardt GA (1995) In vivo assessment of dopamine uptake in rat medial prefrontal cortex: comparison with dorsal striatum and nucleus accumbens, J Neurochem 65:201-207.

Cass WA, Zahniser NR, Flach KA, Gerhardt GA (1993) Clearance of exogenous dopamine in rat dorsal striatum and nucleus accumbens: role of metabolism and effects of locally applied uptake inhibitors. J Neurochem 61:2269-2278.

Cass WA, Manning MW, Dugan MT (1998) Effects of neurotoxic doses of methamphetamine on potassium and amphetamine evoked overflow of dopamine in the striatum of awake rats. Neurosci Lett 248:175-178.

Cumming P, Brown E, Damsma G, Fibiger H (1992) Formation and clearance of interstitial metabolites of dopamine and serotonin in the rat striatum: an in vivo microdialysis study. J Neurochem 59:1905-1914.

Dickinson SD, Sabeti J, Larson GA, Giardina K, Rubinstein M, Kelly MA, Grandy DK, Low MJ, Gerhardt GA, Zahniser NR (1999) Dopamine $\mathrm{D}_{2}$ receptor-deficient mice exhibit decreased dopamine transporter function but no changes in dopamine release in dorsal striatum. J Neurochem 72:148-156.

Eisch AJ, Gaffney M, Weihmuller FB, O’Dell SJ, Marshall JF (1992) 
Striatal subregions are differentially vulnerable to the neurotoxic effects of methamphetamine. Brain Res 598:321-326.

Finnegan KT, Ricaurte G, Seiden LS, Schuster CR (1982) Altered sensitivity to D-methylamphetamine, apomorphine, and haloperidol in rhesus monkeys depleted of caudate dopamine by repeated administration of D-methylamphetamine. Psychopharmacology 77:43-52.

Friedman SD, Castañeda E, Hodge GK (1998) Long-term monoamine depletion, differential recovery, and subtle behavioral impairment following methamphetamine-induced neurotoxicity. Pharmacol Biochem Behav 61:35-44.

Fukumura M, Cappon GD, Pu C, Broening HW, Vorhees CV (1998) A single dose model of methamphetamine-induced neurotoxicity in rats: effects on neostriatal monoamines and glial fibrillary acidic protein. Brain Res 806:1-7.

Garris PA, Walker QD, Wightman RM (1997) Dopamine release and uptake rates both decrease in the partially denervated striatum in proportion to the loss of dopamine terminals. Brain Res 753:225-234.

Gerhardt GA, Oke AF, Nagy G, Moghaddam B, Adams RN (1984) Nafion-coated electrodes with high selectivity for CNS electrochemistry. Brain Res 290:390-395.

Gibb JW, Hanson GR, Johnson M (1994) Neurochemical mechanisms of toxicity. In: Amphetamine and its analogs: psychopharmacology, toxicology, and abuse (Cho AK, Segal DS, eds), pp 269-295. San Diego: Academic.

Glick SD, Dong N, Keller Jr RW, Carlson JN (1994) Estimating extracellular concentrations of dopamine and 3,4-dihydroxyphenylacetic acid in nucleus accumbens and striatum using microdialysis: relationships between in vitro and in vivo recoveries. J Neurochem 62:2017-2021.

Kuczenski R, Segal D (1989) Concomitant characterization of behavioral and striatal neurotransmitter response to amphetamine using in vivo microdialysis. J Neurosci 9:2051-2065.

Lorez H (1981) Fluorescence histochemistry indicates damage of striatal dopamine nerve terminals in rats after multiple doses of methamphetamine. Life Sci 28:911-916.

Luthman J, Friedemann M, Bickford P, Olson L, Hoffer BJ, Gerhardt GA (1993) In vivo electrochemical measurements and electrophysiological studies of rat striatum following neonatal 6-hydroxydopamine treatment. Neuroscience 52:677-687.

McCann UD, Wong DF, Yokoi F, Villemagne V, Dannals RF, Ricaurte GA (1998) Reduced striatal dopamine transporter density in abstinent methamphetamine and methcathinone users: evidence from positron emission tomography studies with $\left[{ }^{11} \mathrm{C}\right] \mathrm{WIN}-35,428$. J Neurosci 18:8417-8422.

Meiergerd SM, Patterson TA, Schenk JO (1993) $\mathrm{D}_{2}$ receptors may modulate the function of the striatal transporter for dopamine: kinetic evidence from studies in vitro and in vivo. J Neurochem 61:764-767.

Melega WP, Raleigh MJ, Stout DB, Huang S-C, Phelps ME (1997a) Ethological and 6-[ $\left.{ }^{18} \mathrm{~F}\right]$ fluoro-L-DOPA-PET profiles of long-term vulnerability to chronic amphetamine. Behav Brain Res 84:259-268.
Melega WP, Raleigh MJ, Stout DB, Lacan G, Huang S-C, Phelps ME (1997b) Recovery of striatal dopamine function after acute amphetamine- and methamphetamine-induced neurotoxicity in the vervet monkey. Brain Res 766:113-120.

Moghaddam B, Bunney BS (1989) Ionic composition of microdialysis perfusing solution alters the pharmacological responsiveness and basal outflow of striatal dopamine. J Neurochem 53:652-654.

O'Callaghan JP, Miller DB (1994) Neurotoxicity profiles of substituted amphetamines in the C57BL/6J mouse. J Pharmacol Exp Ther 270:741-751.

Parsons LH, Schad CA, Justice Jr JB (1993) Co-administration of the $\mathrm{D}_{2}$ antagonist pimozide inhibits up-regulation of dopamine release and uptake induced by repeated cocaine. J Neurochem 60:376-379.

$\mathrm{Pu}$ C, Vorhees CV (1995) Protective effects of MK-801 on methamphetamine-induced depletion of dopaminergic and serotonergic terminals and striatal astrocytic response: an immunohistochemical study. Synapse 19:97-104.

Ricaurte GA, Guillery RW, Seiden LS, Schuster CR, Moore RY (1982) Dopamine nerve terminal degeneration produced by high doses of methylamphetamine in the rat brain. Brain Res 235:93-103.

Robinson TE, Mocsary Z, Camp DM, Whishaw IQ (1994) Time course of recovery of extracellular dopamine following partial damage to the nigrostriatal dopamine system. J Neurosci 14:2687-2696.

Seiden LS, Ricaurte GA (1987) Neurotoxicity of methamphetamine and related drugs. In: Psychopharmacology: the third generation of progress (Meltzer HY, ed), pp 359-366. New York: Raven.

Seiden LS, Fischman MW, Schuster CR (1976) Long-term methamphetamine induced changes in brain catecholamines in tolerant rhesus monkeys. Drug Alcohol Dep 1:215-219.

Suaud-Chagny MF, Dugast C, Chergui K, Msghina M, Ganon F (1995) Uptake of dopamine released by impulse flow in the rat mesolimbic and striatal systems in vivo. J Neurochem 65:2603-2611.

Wightman RM, Zimmerman JB (1990) Control of dopamine extracellular concentration in rat striatum by impulse flow and uptake. Brain Res Rev 15:135-144.

Wilson JM, Kalasinsky KS, Levey AI, Bergeron C, Reiber G, Anthony RM, Schmunk GA, Shannak K, Haycock JW, Kish SJ (1996) Striatal dopamine nerve terminal markers in human, chronic methamphetamine users. Nature Med 2:699-703.

Woolverton WL, Ricaurte GA, Forno LS, Seiden LS (1989) Long-term effects of chronic methamphetamine administration in rhesus monkeys. Brain Res 486:73-78.

Zetterström T, Sharp T, Collin AK, Ungerstedt U (1988) In vivo measurement of extracellular dopamine and DOPAC in rat striatum after various dopamine-releasing drugs; implications for the origin of extracellular DOPAC. Eur J Pharmacol 148:327-334.

Zigmond MJ, Abercrombie ED, Berger TW, Grace AA, Stricker EM (1990) Compensations after lesions of central dopaminergic neurons: some clinical and basic implications. Trends Neurosci 13:290-296. 